ISSN 1816-6075 (Print), 1818-0523 (Online)

Journal of System and Management Sciences

Vol. 10 (2020) No. 3, pp. 35-44

DOI:10.33168/JSMS.2020.0303

\title{
Performance Evaluation according to RSU Range in Vehicle-to-Vehicle Communications
}

\author{
Jin Yeong Um \\ Department of Computer Science \& Engineering, Dongguk University, Seoul, \\ South Korea \\ mog07@dongguk.edu
}

\begin{abstract}
Recently, wireless communication technology and Intelligent Transportation System (ITS) technology in vehicles have been developed. Therefore, it becomes a vehicle and the necessary communication skills for improving safety and traffic flow for drivers has been the study of the Vehicular Ad-hoc Network (VANET) based vehicle communication technology progress. This study aims to implement a simulation of a VANET. It is a special kind of Mobile Ad-hoc Network (MANET) adapted to the communication between vehicles. It moves at high speed and is further classified into vehicle-to-vehicle (V2V) and vehicle-to-infrastructure (V2I) communication methods. To develop a VANET-based vehicle wireless communication technology, it is necessary to establish a test environment and synchronize vehicle mobility in various road scenarios. However, the equipment is expensive for the built experiment environment, and the road traffic situation is difficult to predict. In this study, the simulation of VANET is intended to be tested in an urban environment, and the result can be used also to develop the Media Access Control (MAC) protocols. This paper proposes a scheme for experiments in a similar environment to actual urban traffic and analyzes the performance by dividing it into V2I and V2V nodes according to the Road-Side Unit (RSU) coverage.
\end{abstract}

Keywords: WAVE, VANET, V2I, V2V, RSU

\section{Introduction}

With the recent development of wireless communication technology in vehicles and ITS (Intelligent Transportation System) technology, the necessity of communication technology to improve vehicle and driver safety and traffic flow has emerged. Against this background, research on vehicle communication technology based on Vehicle Ad-hoc Network (VANET) is ongoing (Heidari, E., et al., 2014).

VANET is a special case of Mobile Ad-hoc Network (MANET) where the 
mobile node is a vehicle. The nodes of VANET are highly mobile and tend to follow a systematic path instead of randomly moving. Vehicles not only communicate with each other but also connect to the Road-Side Unit (RSU) and base stations. VANET is expected to support a variety of services such as route planning, traffic alert dissemination, situation information infotainment, and mobile vehicle cloud services (M. W. Ryu, 2010; Uppoor S., and Fiore. M, 2011). Some route planning and monitoring services already in place with VANET systems still have many challenges because of the difficulty in deployment and management (Cooper, C. et al., 2016; Olariu, S. et al., 2011; Singh, S. and Agrawal, S., 2014).

Vehicle to Everything (V2X) is a communication technology that uses wired/wireless networks to exchange information between vehicles and infrastructure. It includes all forms of communication technology between vehicles and various devices on the road. That is, V2X includes vehicle-to-vehicle (V2V), vehicle-to-road infrastructure (V2I, Vehicle to Infra), and vehicle-to-pedestrian (V2P). As vehicle communication may cause mobility problems due to high speed driving, the development of vehicle wireless communication technology based on VANET in consideration of mobility is being actively performed (M. W. Ryu, 2010; Nzouonta, J. et al., 2009). Researches are being conducted on vehicle networks in various fields, such as developing a vehicle framework and developing a model for analyzing the performance of communication technology (JinYeong Um, 2018; Baul Lee and Jin-yeong Um, 2018; JinYeong Um, 2020; Iksoo Kim and Eun-Kyu Lee, 2015; Tonguz, O., 2007).

To develop wireless communication technology with VANET-based vehicles, when the proposed communication technique is tested using a real vehicle, the difference in performance must be tested by a real vehicle. Testbeds of different scenarios must be built on real roads and vehicle mobility synchronized to the scenario. However, it is difficult to experiment according to the scenario because the equipment is expensive, and the road traffic situation is difficult to predict (Uppoor S. and Fiore. M., 2011; Gainaru, A. et al., 2009). The experimental vehicle may cause safety problems (Piorkowski, M. et al., 2008; Martinez, F. J. et al., 2011).

In this paper, we perform a virtual experiment using a network simulator before conducting a large-scale or actual test and use the data obtained to predict the actual test result and present a method to increase the effectiveness of the experiment. The schemes for building a virtual experiment environment using network simulators were presented (JinYeong Um, 2020). However, in the related studies (Uppoor S. and Fiore. M., 2011), it is difficult to analyze the performance of each communication by analyzing the performance of all nodes without considering V2V and V2I communication. This study presents a road-like environment by combining the road traffic simulator Simulation of Urban Mobility (SUMO) which supports the traffic-like environment of the real city, and the Veins framework for VANET wireless communication experiments. In this environment, we present a method for 
analyzing IEEE 802.16 WAVE and performance by classifying V2I and V2V nodes according to RSU (Road Side Units) reception range, rather than performance analysis for all nodes.

It is organized as follows. Chapter 2 introduces of VANET briefly. Chapter 3 describes the structure of VANET Simulator to be used in the experiment in this paper. Chapter 4 explains the simulation topology and operation, Chapter 5 analyzes the experimental results, and Chapter 6 describes the results.

\section{VANET}

In VANET, vehicles communicate with each other via V2V communication in an ad-hoc way and through V2I communication via RSU and mobile broadband (e.g. 4G/LTE). Fig. 1 shows the components and communication with VANET. VANET services include vehicle and road safety services, traffic management services, and infotainment services. Vehicle and road safety services are aimed at reducing traffic accidents and losing human life (Ku, I. et al., 2014)

$\mathrm{V} 2 \mathrm{~V}$ refers to communication between vehicles and is based on IEEE 802.11p and WAVE communications. Each vehicle detects surrounding hazards by exchanging its vehicle information such as the speed and Global Positioning System (GPS), and surrounding situation information through the perception of surrounding conditions while driving. Therefore, V2V communication is a wireless communication technology between vehicles to provide services such as vehicle collision warning to avoid traffic accidents, thereby promoting the safety of vehicle drivers. V2I refers to the communication between the WAVE communication terminal of a vehicle and neighboring base stations. It is a communication method for providing signal information and safety services at intersections, priority signal control for emergency vehicles, and collecting and providing traffic information (IEEE Intelligent Transportation Systems Committee, 2006).

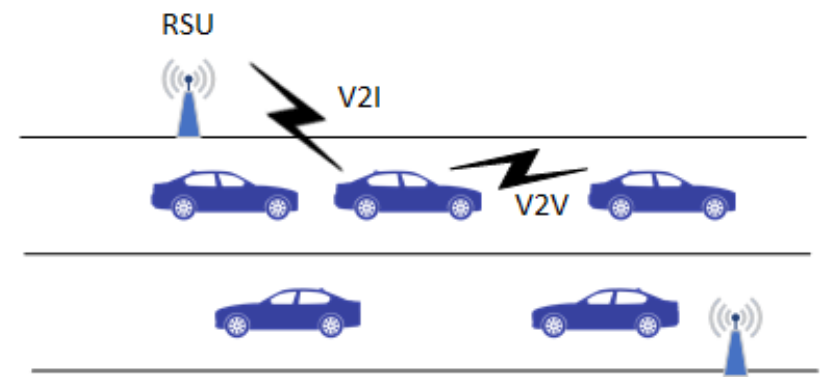

Fig. 1: VANET Communication configuration

\section{VANET Simulator}

Fig. 2 shows the open source framework which performs VANET simulation by connecting network simulator using Objective Modular Network Testbed in C++ $(\mathrm{OMNeT}++)$ and Veins, a road traffic simulation. OMNeT ++ is an object-oriented 
event-based simulator engine and an open source simulation program that can be used for various types of simulations, including communication protocols, computer networks, multiprocessors, and distributed systems. There is a lack of connectivity and mobility to simulate a fast-moving vehicle network (Noori H., 2012; A. Kopke et al., 2008; OMNET++). Mixed Simulator (MiXiM) is an OMNeT++ modeling framework created for mobile and fixed wireless networks (e.g. Wireless Sensor Networks (WSN), Body Area Networks (BAN), ad-hoc networks, vehicular networks, etc.). It offers detailed models of radio wave propagation, interference estimation, radio transceiver power consumption, and wireless Media Access Control (MAC) protocols (A. Kopke et al., 2008; MiXiM).

SUMO is a free and open traffic simulation suite. It allows modeling of intermodal traffic systems including road vehicles, public transport, and pedestrians. SUMO is also included with various supporting tools that can handle different tasks, such as route finding, visualization, network import, and emission calculation. It can further be enhanced with custom models and provides various features to remotely control the simulation SUMO has been widely used to tackle with variety of research project, for example evaluating modern algorithm in increasing the performance of traffic lights (Kit Guan Lim et al., 2016)

Veins were developed based on MiXiM. It runs two simulators in parallel over SUMO and OMNeT ++ via Transmission Control Protocol (TCP) sockets and receives information about vehicle generation and mobility from SUMO, allowing each vehicle to be recognized as a node in OMNeT ++ to enable VANET communication simulation. Supporting road traffic, the SUMO simulator supports road networks including roads, streets, traffic lights, intersections, and details such as car generation, speed, and direction of each car. The starting point and destination, time, and location can be displayed, and maps can be extracted from OpenStreetMap to provide an environment similar to the road traffic in actual cities (Uppoor S. and Fiore. M., 2011; Behrisch, M. et al., 2011; Krajzewicz, D., 2002).

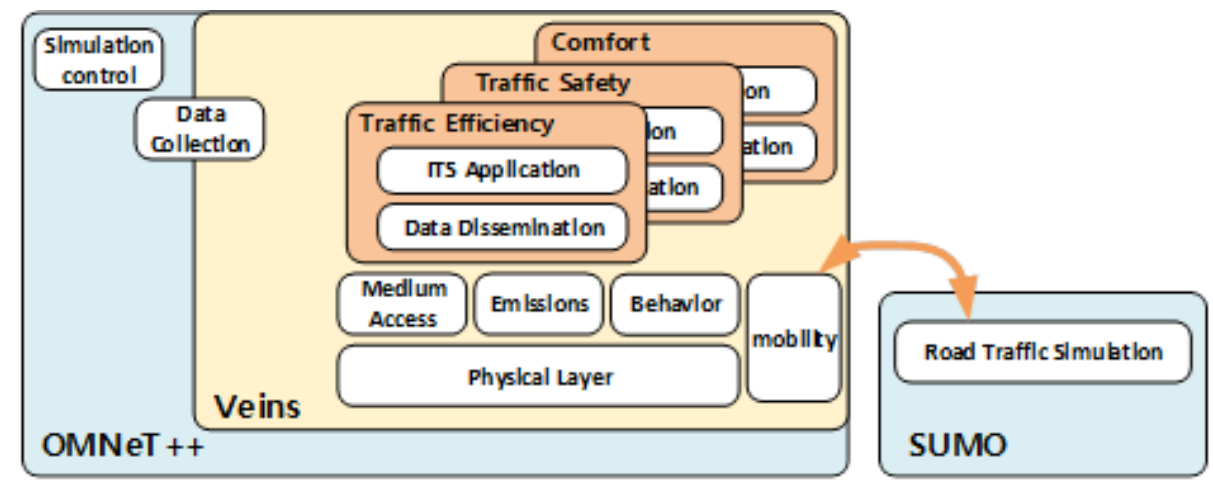

Fig. 2: Framework of OMNet++, Veins, and SUMO 


\section{VANET Experiment Environment for RSU Receiving Range Control}

Housing is one among the essential needs of person and Government has the prime duty to supply affordable housing for all income groups. During this regard, Government has got to take adequate measures for policy implication that specialize in the middle-income group in terms of budgeting, loans from provident funds, pension etc. If these provisions were made, several real estate loan opportunities will rise easing the financial requirements of housing for middle-income group. Public sector should undertake a greater number of site services scheme for exploitation and will work ashore policy and land price mechanism. For instance, in Sonadanga area most of the serviced plots are acquired by higher-middle income group. If certain number of small plots (2-3 katha sized) were developed and facilities were provided considering the middle-middle and lower-middle income group then they also could easily afford those plots. So, Government should develop land considering people of specific income group to supply affordable housing for all people. On the opposite hand, formal private sector in Khulna City isn't yet developed properly due to lack of reliance on the developers and lack of organizations among the individual owners just in case of co-operatives. Government can take initiative during this regard providing an organizational body for funding and monitoring the works of personal formal sector. By this, more people are going to be ready to afford housing by raising funds for initial deposit requirement and can also believe the standard and facilities provided by the developers.

Uppoor S. and Fiore. M. (2011) conducted a study on vehicle traffic for 24 hours in $400 \mathrm{~km} 2$ of $\mathrm{K}^{*} \mathrm{oln}$, in Germany. Without considering $\mathrm{V} 2 \mathrm{~V}$ and V2I communication separately, there is a disadvantage that performance analysis for each communication cannot be performed by analyzing the performance of all communication nodes. It is not possible to perform performance analysis when developing a communication protocol of a vehicle network in which heterogeneous networks are mixed.

This paper considered a simple topology, as shown in Fig. 3 to classify V2V and V2I. In the said topology, all nodes communicating are divided into I-RNR (Vehicles within RSU's communication range) and Non-RNR (Vehicles outside the RSU's range of communication) located on the roadside to calculate the number of successful transmissions of I-RNR and Non-RNR. I-RNR means to communicate with V2I, while Non-RNR means to communicate with V2V.

The operation process of each node in the topology is as follows. For reference, Veins communicates based on the vehicle node information received from SUMO through a TCP socket.

1) Each node including RSU performs channel switching.

2) If a beacon event occurs during channel switching, broadcast a beacon 
message to nodes within the Beacon range.

3) If the transmitting node is within the reception range of the RSU, packets are transmitted from the RSU and all nodes within the transmission range to the target node. If it is not within the reception range of the RSU, it communicates between the vehicles $(\mathrm{V} 2 \mathrm{~V})$ through communication between all nodes in the transmission range except the RSU and transmits a packet to all nodes in the reception range.

4) If the packet transmission is completed and the simulation end time, the process ends. If the simulation end time is not, proceed to step 1) again.

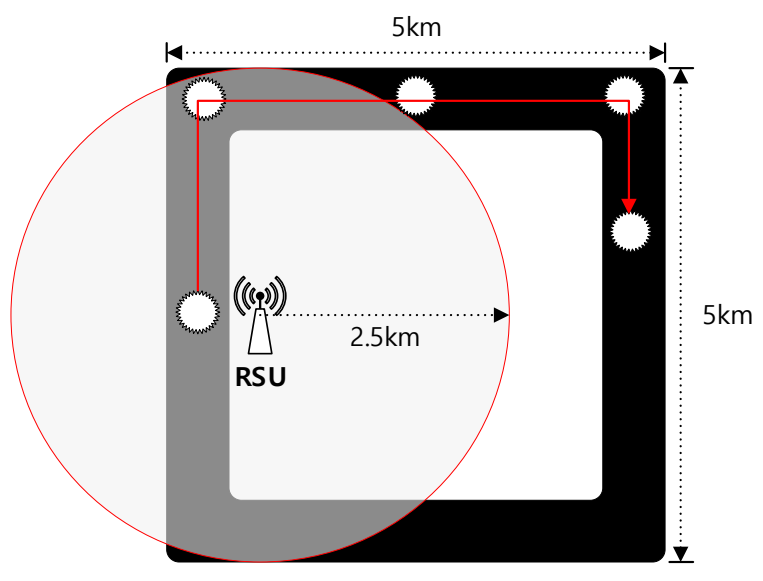

Fig. 3: I-RNR and Non-RNR Simple Topology

\section{Performance Analysis}

In the scenario described in Section 4, I-RNR and Non-RNR are separated to. They enter the I-RNR communication range (or non-RNR communication range) for the entire experiment time. After selecting one node among all nodes, it calculates the number of beacon transmissions for all the adjacent nodes. The beacon transmission probability during the experiment time is calculated using the number of beacon transmissions of all nodes and the number of beacons transmitted by the selected node. A road topology consisting of SUMO is used, as shown in Fig. 3, and the speed of each vehicle node is limited to $15 \sim 70 \mathrm{~km}$. A new vehicle node was generated every second. Therefore, as time passes, the number of neighboring nodes increases, so the total number of beacon transmissions increases and the experiment environment is configured to increase the probability of collisions.

Table 1 shows the experimental parameters, beacon size, beacon transmission bandwidth, minimum signal attenuation threshold, and Constant Bit Rate (CBR) and maximum transmission power parameter values.

Before executing this experiment, since the coordinator exists when the RSU is within the reception range, it was expected that the beacon transmission probability would be higher than where the RSU was not included in the RSU reception range. 
However, as shown in Figure 4, unlike the previous idea, the probability of beacon transmission of nodes not in the RSU reception range was higher than the probability of beacon transmission of nodes belonging to the RSU reception range. Although RSU is a coordinator, beacon continues to be generated as a node, causing a problem where the beacon transmission delay time increases. In terms of distance, the beacon message transmission delay time is shorter because the beacon message is transmitted and received between the vehicle and the nearby vehicle when communicating between vehicles compared to vehicles communicating with a remote RSU.

Table 1: Experimental Parameter

\begin{tabular}{|c|c|}
\hline Parameter & value \\
\hline Beaconing rate & $10 \mathrm{~Hz}$ \\
\hline Beacon size & $256 \mathrm{bytes}$ \\
\hline Minimum signal attenuation threshold & $-89 \mathrm{dBm}$ \\
\hline Maximum transmission power & $20 \mathrm{~mW}$ \\
\hline
\end{tabular}

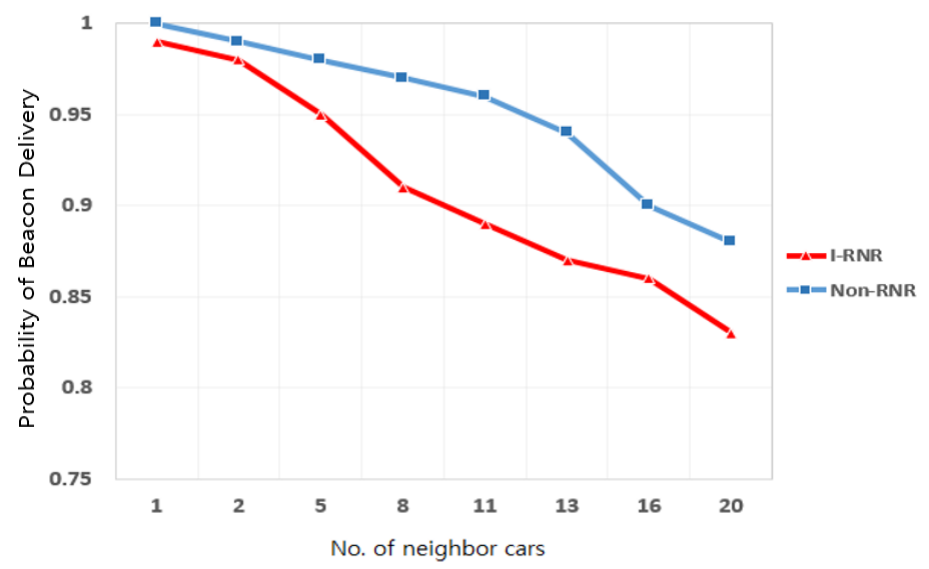

Fig. 4 I-RNR and Non-RNR Beacon Transmission Probabilities

\section{Conclusion}

In this paper, we performed a virtual experiment using a network simulator before conducting a large-scale or actual test and used the data obtained to predict the actual test result and presented a method to increase the effectiveness of the experiment. Existing research has suggested ways to build a virtual experiment environment using network simulators. However, it was difficult to analyze the performance of each communication by analyzing the performance of all nodes without considering $\mathrm{V} 2 \mathrm{~V}$ and $\mathrm{V} 2 \mathrm{I}$ communication. We constructed a road-like 
environment by combining the road traffic simulator SUMO, which supports a traffic-like environment in the real city, and the Veins framework for VANET wireless communication experiments. Using this environment, we analyzed the performance of classification into $\mathrm{V} 2 \mathrm{I}$ and $\mathrm{V} 2 \mathrm{~V}$ nodes according to the RSU reception range, and not by performance analysis for all nodes. This study confirmed that vehicles communicating outside the RSU receiving range have a higher probability of beacon transmission than vehicles existing in the RSU receiving range. It is expected that it is suitable to communicate with $\mathrm{V} 2 \mathrm{~V}$ in a high vehicle density section and V2I in a low vehicle density section.

Future research will have to analyze and experiment with the appropriate RSU placement distances for placing RSUs in high-density vehicles, such as intersections and highways, and where they are not. Even if the RSU exists, improving the throughput and reliability by switching to $\mathrm{V} 2 \mathrm{~V}$ communication according to vehicle density must be studied.

\section{Acknowledgments}

This research was supported by Basic Science Research Program through the National Research Foundation of Korea (NRF) funded by the Ministry of Education (No. 2017R1D1A1B03032736).

\section{References}

Kopke A., Swigulski M., Wessel K., Wilkomm D., Haneveld PT, Parker TEV, OWVisser, Lichte HS and Valentin S. (2008). Simulation wireless and mobile networks in OMNET++ the MiXiM vision. In Proceedings of the 1st international conference, 1-8.

Baul Lee and Jin-yeong Um. (2018). Extended IEEE 802.11p based on Distance in Automotive Systems. International Journal of Science and Engineering for Smart Vehicles, 2 (2), 13-18.

Behrisch, M., Bieker, L., Erdmann, J., and Krajzewicz, D. (2011). SUMO simulation of urban mobility: an overview. The Third International Conference on Advances in System Simulation.

Cooper, C., Franklin, D., Ros, M., Safaei, F., \& Abolhasan, M. (2016). A comparative survey of VANET clustering techniques. IEEE Communications Surveys \& Tutorials, 19(1), 657-681.

Gainaru, A., Dobre, C. and Cristea, V. (2009). A realistic mobility model based on social networks for the simulation of VANETs. In VTC Spring 2009-IEEE 69th Vehicular Technology Conference, 1-5. 
Hasson, S.T. and Zahraa Y.H. (2017). Roads clustering approach's in VANET models. 2017 Annual Conference on New Trends in Information \& Communications Technology Applications (NTICT).

Heidari, E., Gladisch, A., Moshiri, B., \& Tavangarian, D. (2014). Survey on location information services for Vehicular Communication Networks. Wireless networks, 20(5), 1085-1105.

IEEE Intelligent Transportation Systems Committee. (2006). IEEE Trial-Use Standard for Wireless Access in Vehicular Environments (WAVE)-Multi-Channel Operation. IEEE Std, 1609-4.

Iksoo Kim and Eun-Kyu Lee. (2015). Framework for Safety Driving System in Connected-Vehicle Communication Networks. International Journal of Multimedia and Ubiquitous Engineering, 10 (10), 137-146.

JinYeong Um. (2020). Performance Analysis According to RSU Range of VANETbased Communication Vehicle. International Journal of Science and Engineering for Smart Vehicles, 4 (1), 1-6.

JinYeong Um (2018). A Scheme for Real-time Data Control in Automotive Systems. International Journal of Science and Engineering for Smart Vehicles, 2 (2), 7-12.

JinYeong Um (2020). A Performance Model of Controller Area Networks for Vehicles under Unsaturated Traffic. International Journal of Reliable Information and Assurance, 8 (1), 1-6.

Kit G.L., Chun H.L., Renee K.Y.C., Kiam B. Y., and Kenneth T.K.T. (2016). Simulators for vehicular ad hoc network (VANET) development. IEEE International Conference on Consumer Electronics-Asia (ICCE-Asia), 1-4.

Krajzewicz, D., Hertkorn, G., Rössel, C. and Wagner, P. (2002). SUMO (Simulation of Urban MObility) - an open-source traffic simulation. 4th middle East Symposium on Simulation and Modelling (MESM), 183-187.

Ku, I., Lu, Y., Gerla, M., Gomes, R. L., Ongaro, F., and Cerqueira, E. (2014). Towards software-defined VANET: Architecture and services. In 2014 13th annual Mediterranean ad hoc networking workshop (MED-HOC-NET), 103-110.

Lochert, C., Scheuermann, B., Wewetzer, C., Luebke, A., and Mauve, M. (2008). Data aggregation and roadside unit placement for a vanet traffic information system. In Proceedings of the fifth ACM international workshop on VehiculAr InterNETworking, 58-65.

Martinez, F. J., Toh, C. K., Cano, J. C., Calafate, C. T. and Manzoni, P. (2011). A survey and comparative study of simulators for vehicular ad hoc networks (VANETs). Wireless Communications and Mobile Computing, 11(7), 813-828. 
MiXiM, http://mixim.sourceforge.net/

Ryu, M. W., Cha, S. H. and Cho, K. H. (2010). An Enhanced Greedy Message Forwarding Protocol for Increasing Reliability of Mobile Inter-Vehicle Communication. The Institute of Electronics Engineers of Korea, 47 (4), 43-50.

Noori H., (2012). Realistic Urban Traffic Simulation as Vehicular Ad-hoc Network(VANET) via Veins Framework.

Nzouonta, J., Rajgure, N., Wang, G. and Borcea, C. (2009). VANET routing on city roads using real-time vehicular traffic information. IEEE Transactions on Vehicular technology, 58(7), 3609-3626.

Olariu, S., Khalil, I. and Abuelela, M. (2011). Taking VANET to the clouds. International Journal of Pervasive Computing and Communications.

OMNET++, https://omnetpp.org/

Piorkowski, M., Raya, M., Lugo, A. L., Papadimitratos, P., Grossglauser, M., and Hubaux, J. P. (2008). TraNS: realistic joint traffic and network simulator for VANETs. ACM SIGMOBILE mobile computing and communications review, 12(1), 31-33.

Singh, S., and Agrawal, S. (2014). VANET routing protocols: Issues and challenges. In 2014 Recent Advances in Engineering and Computational Sciences (RAECS), 1-5.

Tonguz, O., Wisitpongphan, N., Bait, F., Mudaliget, P., and Sadekart, V. (2007). Broadcasting in VANET. In 2007 mobile networking for vehicular environments, 712.

Uppoor S. and Fiore. M., (2011). Large-scale urban vehicular mobility for networking research. Vehicular Networking conference(VNC), 62-69.

Veins, https://veins.car2x.org/documentation/ 